\title{
CULTURA TRIBUTARIA EN HERMOSILLO, SONORA MÉXICO
}

\author{
María del Carmen Morales Tostado', Leivy Gisel Echeverría Rodríguez², \\ Beatriz Elena Huerta Urquijo ${ }^{3}$, Erika Lorena Quinto Lorenzo ${ }^{4}$
}

SUMARIO: I. Introducción. II. Desarrollo. III. Conclusiones IV.Bibliografía

\section{RESUMEN}

La cultura tributaria es un referente importante en el contexto gubernamental, en virtud de que crea conciencia a los ciudadanos para cumplir con sus obligaciones fiscales.En algunos estudios se señala que en México la evasión de impuestos alcanza el $2.6 \%$ del PIB, a pesar que se han implementado estrategias para impulsar la cultura fiscal, argumentan el mal uso que se les da por parte de sus gobernantes. El presente artículo, tiene por objetivo, identificar la cultura fiscal que poseen los contribuyentes en Hermosillo Sonora y proponer acciones para mejorar los valores, creencias y actitudes acerca de la tributación.Dentro de la estrategia metodológica de la investigación, se puede compartir que es de tipo descriptivo, para recolección de datos se diseñó una encuesta de Cultura Fiscal que está conformada por 5 bloques de preguntas de opción múltiple. Participaron los contribuyentes que acudieron al módulo de Servicio al Contribuyente del Servicio de Administración Tributaria de Hermosillo Sonora y se aplicaron 300 encuestas. Como parte de los resultados, se conoce que un alto porcentaje de los contribuyentes encuestados presenta bajo conocimiento en relación a los

\footnotetext{
1 Docente Investigadora del Departamento de Contabilidad, Coordinadora de la Maestría en Fiscalización y Control Gubernamental, Universidad de Sonor, correo electrónico: carmen.morales@unison.mx

2 Egresada de la Maestría en Impuestos de la Universidad de Sonora, correo electrónico: gisel.echeverria09@gmail.com

${ }_{3}$ Profesora del Departamento de Contabilidad de la Universidad de Sonora, correo electrónico: beatriz.huerta@unison.mx.

${ }^{4}$ Estudiante de la Maestría en Fiscalización y Control Gubernamental de la Universidad de Sonora, correo electrónico: erika_quint@hotmail.com
} 


\section{Cultura tributaria en Hermosillo, Sonora, México}

Año 11, Número 21 julio-diciembre de 2019

María del Carmen Morales Tostado, Leivy Gisel Echeverría Rodríguez, Beatriz Elena Huerta Urquijo, Erika Lorena Quinto Lorenzo

impuestos que están obligados a contribuir, desconoce el destino de los impuestos recaudados por el gobierno, es importante destacar que un $73 \%$ de los encuestados coincide que la educación fiscal puede generar conciencia entre los ciudadanos para que cumplan con sus obligaciones fiscales.

\section{PALABRAS CLAVE: Tributación, cultura fiscal y legalidad}

\section{TAX CULTURE IN HERMOSILLO, SONORA MEXICO}

\section{ABSTRACT}

The tax culture is an important reference in the government context, because it creates awareness for citizens to meet their tax obligations. Some studies indicate that in Mexico tax evasion reaches $2.6 \%$ of PIB, although strategies have been implemented to boost fiscal culture, they argue that they are misused by their rulers. The purpose of this article is to identify the tax culture that taxpayers possess in Hermosillo Sonora and propose actions to improve values, beliefs and attitudes about taxation. Within the methodological strategy of the investigation, it can be shared that it is descriptive, for the purpose of data collection, a Fiscal Culture survey was designed consisting of 5 blocks of multiple choice questions. Taxpayers who attended the Taxpayer Service module of the Tax Administration Service of Hermosillo Sonora participated and 300 surveys were applied. As part of the results, it is known that a high percentage of the taxpayers surveyed have low knowledge in relation to the taxes they are required to contribute, the destination of the taxes collected by the government is unknown, it is important to highlight that $73 \%$ of the Respondents agree that tax education can raise awareness among citizens to meet their tax obligations.

KEY WORDS: Taxation, tax culture and legality 


\section{INTRODUCCIÓN}

En merecido homenaje- póstumo a Don Emilio Margáin Manautou reconocido administrativista y fiscalista, autor de extensa obra en las ramas del Derecho Administrativo y del Derecho Fiscal, legado invaluable para los estudiosos de las ciencias jurídico, administrativa y contable, va nuestra presente colaboración, en coincidencia, especialmente con una de sus obras "Nociones de Política Fiscal" 5 en cuanto a la responsabilidad que corresponde al Fisco de contribuir a la educación fiscal del contribuyente, tal y como en el presente artículo se describe de manera sucinta la situación de la educación fiscal en México y como percibe la sociedad actual la cultura tributaria, esto con el fin de conocer que tanto influye en la conducta actual de los ciudadanos en cuanto a la tributación.

Para ello, es necesario señalar lo que se entiende por cultura fiscal, considerada como una conducta intrínseca manifestada por la ciudadanía con relación a sus responsabilidades y valores éticos al momento de cumplir con sus obligaciones fiscales.

La cultura tributaria por su parte es la manera en que la ciudadanía percibe su responsabilidad al momento de cumplir o no con sus obligaciones fiscales, conforme al grado de satisfacción que le brinde el Estado a través de los servicios públicos ${ }^{6}$, la consecución de esta cultura tributaria es el resultado de una educación fiscal.

Pero ¿qué sucede con la cultura tributaria en México? ¿Es consciente la ciudadanía de la importancia de una cultura tributaria?

\footnotetext{
${ }^{5}$ Margáin Manautou, Emilio, Nociones de Política Fiscal, 2ạ. Ed., México,Porrúa,1998,p.85

${ }^{6}$ Escalante G. La Cultura Tributaria de los Contribuyentes. Revista del Especialización para ejecutivos. 2015. P. 44-47.
} 


\section{Cultura tributaria en Hermosillo, Sonora, México}

Año 11, Número 21 julio-diciembre de 2019

María del Carmen Morales Tostado, Leivy Gisel Echeverría Rodríguez, Beatriz Elena Huerta Urquijo, Erika Lorena Quinto Lorenzo

A grandes rasgos pareciera que no pasa nada, sin embargo, sus efectos son contraproducentes en un país donde la evasión fiscal es una práctica común, puesto que de acuerdo con la investigación de Escalante 7 de cada 10 personas tienen una escasa cultura tributaria.

Lo anterior es consecuencia de una omisión sobre educación fiscal en la formación de cualquier estudiante, pues en necesario que todo ciudadano conozca sus derechos y obligaciones.

La Secretaría de Hacienda y Crédito Público (SHCP) con ayuda de uno de sus órganos ha implementado programas que intentan concientizar a la ciudadanía sobre la importancia de tener una cultura fiscal, ejemplo de ello fue el Programa Nacional de Civismo. Sin embargo, además de no haberse implementado en todo el país, no se le dio continuidad, es decir son esfuerzos que no se terminan por concretar.

También, hay que recordar que no todo es responsabilidad de una sola Secretaría de Estado, dado que la poca legitimidad y la nula credibilidad de los gobiernos sobre el manejo de los recursos públicos, los numerosos casos conocidos de corrupción, ha fomentado de manera indirecta la antipatía por parte de los ciudadanos.

En este sentido, es importante compartir lo planteado por Nieto, quien expresa lo siguiente:

En nuestro país, no es ningún secreto, la mayoría de la gente tiene un cierto recelo al pago de impuestos, no solo porque nunca se ha tenido la educación tributaria como parte de la formación cívica, que debería tener cada uno de los ciudadanos, sino porque a través del tiempo, tanto las autoridades encargadas de administrar los impuestos como los servidores públicos, se han visto beneficiados con grandes cantidades de dinero, provenientes de actos ilícitos dentro de su administración?

\footnotetext{
${ }^{7}$ Nieto, Salvador. La educación tributaria como solución a la baja recaudación fiscal en México. Guanajuato, Guanajuato, México. 2003, p1.2.
} 
Este estudio, nos permite afirmar que la educación es una vez más, la manera de prevenir los diversos problemas que aquejan a nuestro país, por ello, Nieto propone que estos temas se deberían de tocar en los libros de texto en educación básica, a fin de promover la "cultura tributaria", de esta manera al llegar al nivel de educación superior, tendrían una formación estructurada y afianzada en cuanto a lo que implica una educación cívica-fiscal, puesto que el beneficio de unos no siempre implica el bien de la mayoría.

Así mismo, resulta necesario que la Secretaría de Hacienda y Crédito Público (SHCP) a través del Sistema de Administración Tributaria (SAT) divulgue el manejo y uso de los impuestos y las instancias de asesoría que coadyuven al cumplimiento de la ciudadanía para con el Estado. De igual manera, se debe implementar un programa de civismo fiscal permanente y de forma constante para de esta manera visualizar resultados en cuanto al pago voluntario de impuestos.

\section{DESARROLLO}

México cuenta con un Programa Nacional de Educación Fiscal, pero no es una política de Estado permanente para despertar la conciencia ciudadana del pago de impuestos ${ }^{8}$. Por lo que es necesaria una coordinación entre las distintas Universidades del país, la participación de las pequeñas y medianas empresas (Pymes), emprendedores y personal de gobierno, al fin de crear una sinergia en quienes formamos parte de esta sociedad con el objetivo de establecer una cultura tributaria.

Se necesita comenzar por construir y reestructurar la cultura tributaria de la sociedad, para que ésta cumpla con sus obligaciones fiscales con confianza y certidumbre del bienestar económico social que representa el contribuir al gasto público del país.

\footnotetext{
${ }^{8}$ Flores, Leonor, México, sin educación fiscal: OCDE. El Universal, 28 de julio, sección Economía,2015 [en línea] Disponible en: https://www.eluniversal.com.mx/articulo/cartera/economia/2015/07/28/mexico -sin-educacion-fiscal-ocde(consutado el 12 de septiembre de 2019)
} 


\section{Cultura tributaria en Hermosillo, Sonora, México}

Año 11, Número 21 julio-diciembre de 2019

María del Carmen Morales Tostado, Leivy Gisel Echeverría Rodríguez, Beatriz Elena Huerta Urquijo, Erika Lorena Quinto Lorenzo

La Constitución Política de los Estados Unidos Mexicanos (CPEUM) establece como obligación de los mexicanos lo siguiente:

Son obligaciones de los mexicanos Contribuir para los gastos públicos, así de la Federación, como de los Estados, de la Ciudad de México y del Municipio en que residan, de la manera proporcional y equitativa que dispongan las leyes (CPEUM, artículo 31, fracción IV).

En este artículo encontramos los cinco principios constitucionales en materia de derechos humanos, mismos que toda autoridad en el ámbito de sus competencias deberá respetar, por ello y en materia fiscal estos principios se expresan de la siguiente manera:

Principio de legalidad, establece que todo acto que emane de las autoridades en todos sus niveles debe estar fundado en un ordenamiento legal. Es decir, estamos obligados a respetar lo establecido en la CPEUM y en las leyes en cuanto a nuestros deberes fiscales.

Por otra parte, el principio de proporcionalidad menciona esencialmente que los sujetos pasivos de la relación jurídica tributaria deben contribuir al gasto público en función de su respectiva capacidad contributiva, debiendo aportar una parte adecuada de sus ingresos, utilidades, rendimientos o la manifestación de riqueza gravada.

El principio de proporcionalidad se entiende, como la contribución a los gastos públicos en cualquier espacio territorial del que se forme parte, federal, estatal o municipal, como cumplimiento a las leyes tributarias, de acuerdo con la normativa vigente y conforme lo establecido, en función de su capacidad económica y al costo en las demás cargas fiscales.

Por su parte, el principio de equidad significa la igualdad ante la misma ley tributaria de todos los sujetos pasivos de un mismo tributo, los que en tales condiciones deben recibir un tratamiento idéntico en lo concerniente a 
hipótesis de causación, acumulación de ingresos gravables, deducciones permitidas, plazos de pago, etcétera ${ }^{9}$.

Y por último tenemos el principio de destino al gasto público, el cual se refiere a las erogaciones que realiza el Estado a través de los tres poderes y los tres niveles de gobierno, cuya finalidad es dar cumplimiento al artículo 25 de la CPEUM, el cual en su primer párrafo establece que es obligación del Estado, mediante la competitividad, el fomento del crecimiento económico y el empleo; una justa distribución del ingreso y la riqueza para tal efecto existe un marco jurídico para que tales postulados se cumplan.

Este principio se apoya del Código Fiscal de la Federación en su artículo 1ํe el cual menciona lo siguiente:

"Las personas físicas y las morales están obligadas a contribuir para los gastos públicos conforme a las leyes fiscales respectivas. Las disposiciones de este Código se aplicarán en su defecto y sin perjuicio de lo dispuesto por los tratados internacionales de los que México sea parte. Sólo mediante ley podrá destinarse una contribución a un gasto público específico". ${ }^{10}$

Las obligaciones deben ser de conocimiento de los ciudadanos ya que se encuentran establecidas en nuestra Constitución Política y en las leyes tributarias.

Cabe mencionar, algunas iniciativas y programas realizados por parte de la Secretaria de Hacienda a través del SAT para el aumento de la recaudación de impuestos, con el fin de apoyar la actividad económica del país, además de facilitar este ejercicio para aquellos deudores que han evadido esta responsabilidad o bien no tienen una correcta estrategia fiscal, misma que los ha llevado a incumplir su encargo.

\footnotetext{
${ }^{9}$ Proporcionalidad y equidad tributarias establecidas en el artículo 31, fracción IV, Constitucional., https://sjf.scjn.gob.mx/sjfsist/paginas/DetalleGeneralV2.aspx?id=232309\&Clase=DetalleTesis BL (consultada el 26 de septiembre de 2019).

${ }^{10}$ Código Fiscal de la Federación (México: Cámara de Diputados del H. Congreso de la Unión, 2019) artículo 1 primer párrafo.
} 


\section{Cultura tributaria en Hermosillo, Sonora, México}

Año 11, Número 21 julio-diciembre de 2019

María del Carmen Morales Tostado, Leivy Gisel Echeverría Rodríguez, Beatriz Elena Huerta Urquijo, Erika Lorena Quinto Lorenzo

Algunas de estas iniciativas han sido, el "Programa de Apoyo a Deudores del Fisco" implementado por el SAT en 1996, año en el que el país atravesaba por ciertos problemas liquidez en diversos sectores económicos y cuyo objetivo era el apoyar la actividad económica del país y el cumplimiento de las obligaciones fiscales de los contribuyentes, el Ejecutivo Federal expidió el Decreto que establecía dicho programa. Mediante este programa se otorgó una condonación parcial de los adeudos por impuestos y/o multas federales derivados del incumplimiento de disposiciones fiscales federales, sus actualizaciones y accesorios, a los contribuyentes que se unieran al programa.

Por otra parte, tenemos, el "Programa de Actualización y Registro" (PAR) el cual estaba orientado motivar el cumplimiento voluntario, combatir la evasión, el contrabando y la informalidad, eficientar la administración tributaria, contar con una organización integrada que sea reconocida por su capacidad, ética y compromiso.

EI PAR era un programa estratégico del SAT para mantener al día la base de contribuyentes, que responde a la demanda de la sociedad para que se actualice y amplíe la base de contribuyentes, sea más eficiente la operación recaudatoria, equitativa la recaudación y la población reciba mayores beneficios sociales (SAT 2008).

Los objetivos del SAT con este programa fueron de gran interés ya que buscaba promover la cultura tributaria sensibilizando a los futuros ciudadanos del país sobre la importancia de contribuir, brindando así facilidades a los contribuyentes para que tramiten o actualicen su registro federal de contribuyentes (RFC), también busca inscribir a contribuyentes omisos en este registro para fomentar el cumplimiento oportuno de las obligaciones fiscales.

Así mismo, una iniciativa implementada por el Gobierno de la Republica para impulsar la economía de las familias mexicanas y su desarrollo al integrarlas a la formalidad, fue "Crezcamos Juntos". La finalidad era que, al unirse a esta iniciativa, se ingresara a la formalidad ante el SAT sin pagar 
impuestos durante el primer año, y con importantes descuentos durante nueve años más.

Dicho programa fue impulsado desde comienzos del año 2014, debido a la derogación del pasado régimen para personas físicas que se encontraban en el mismo supuesto, el Régimen de Pequeños Contribuyentes (REPECOS).

El Régimen de Incorporación Fiscal ${ }^{11}$, se enfoca en una especie de transitoriedad en la cual se puede reducir ISR y se goza de una tasa preferencial del IVA y el Impuesto Especial sobre Productos y Servicios (IEPS).

Aparte de la reducción en la sobre el ISR, otros beneficios del Régimen de Incorporación Fiscal es el referente a la salud y seguridad social, en el cual existe una reducción del $50 \%$ en el pago de cuotas de seguridad social, el cual disminuye gradualmente un $10 \%$ cada dos años.

Otro beneficio es el crédito para vivienda, el cual aplica para los trabajadores de los patrones, con un subsidio gradual en el pago de aportaciones que disminuye un $10 \%$ cada 2 años.

Como cuarto beneficio se presenta la posibilidad del micro-empresario de acceder a financiamiento y apoyo para su negocio, ya que como empresas integradas a la economía formal es más sencillo ofrecer una garantía de pago a la institución de crédito.

Un beneficio más del régimen es la simplicidad con la que pueden determinar sus contribuciones, además de la facilidad con la que pueden presentar sus declaraciones, lo anterior en el sentido de que las declaraciones de pagos en negocios dedicados únicamente a la venta de alimentos o medicinas se presentan en momentos distintos que las de las personas morales incorporadas al régimen general de contribuyentes; de conformidad con la Ley ISR las personas ubicadas en dicho régimen deberán presentar sus pagos definitivos en materia del ISR de manera bimestral en los meses de

\footnotetext{
${ }^{11}$ Gobierno de México, portal único, Régimen de Incorporación Fiscal "Beneficios", Secretaria de Hacienda y Crédito Público. http://omawww.sat.gob.mx/RegimenDelncorporacionFiscal/beneficios.htm (consultada el 15 de octubre de 2019).
} 


\section{Cultura tributaria en Hermosillo, Sonora, México}

Año 11, Número 21 julio-diciembre de 2019

María del Carmen Morales Tostado, Leivy Gisel Echeverría Rodríguez,

Beatriz Elena Huerta Urquijo, Erika Lorena Quinto Lorenzo

marzo, mayo, julio, septiembre, noviembre y enero; por lo que se facilita tangiblemente el cumplimiento de obligaciones fiscales.

Además, se presenta la posibilidad de participación en múltiples programas federales para promover el régimen y la facilitación para los contribuyentes de crecer, cumplir y pagar impuestos.

Ahora en apoyo a lo anterior se originó el programa SUMATE el cual básicamente tiene los mismos objetivos, el incorporar a la formalidad a aquellas personas que realizan actividades económicas sin estar inscritos en el RFC y apoya en el cumplimiento voluntario, correcto y oportuno de las obligaciones fiscales. Y con ello contribuir al crecimiento económico del Estado que garantice el desarrollo igualitario, incluyente y sostenible para todos.

Son varios los beneficios y facilidades con las que cuenta y apoya el SAT para incentivar la incorporación Fiscal. Sin embargo, esto no significa que en su mayoría las personas se sientas motivadas a cumplir con sus obligaciones tributarias, además de la escasa educación fiscal y el desinterés en cuanto a recibir educación cívica que influya en estas obligaciones.

\section{RESULTADOS}

Dentro de la estrategia metodológica de la investigación, se eligió el enfoque cualitativo, de tipo descriptivo y para recolección de datos se diseñó una encuesta sobre Cultura Fiscal conformada por 5 bloques de preguntas de opción múltiple con escala tipo Likert y se presenta como Anexo 1.

Se incluyeron todos aquellos contribuyentes que acudieron al módulo de Servicio al Contribuyente del Servicio de Administración Tributaria de Hermosillo Sonora y se aplicaron 300 encuestas.

A continuación se presenta de manera resumida y en gráficas parte de los resultados de la investigación.

La siguiente gráfica muestra el resultado de la respuesta a la pregunta: ¿Conoce los impuestos que como ciudadano está obligado a contribuir? 
Gráfica 1: Conocimiento de los impuestos obligado a contribuir

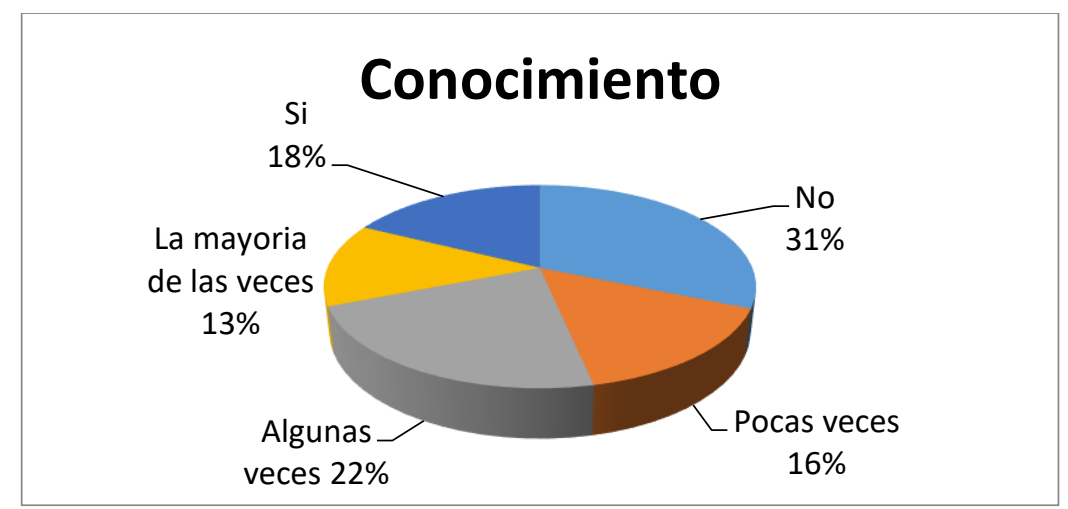

Fuente: Elaboración propia con datos del cuestionario para la evaluación de la cultura fiscal

Se puede observar que un alto porcentaje de los contribuyentes encuestados presenta bajo conocimiento en relación a los impuestos que están obligados a contribuir, esta conducta de desconocimiento resulta de la falta de educación fiscal, siendo esto fundamental a desarrollar el incumpliendo de las obligaciones fiscales o el uso incorrecto de las leyes fiscales, especialmente la credibilidad del ciudadano para contribuir al gasto público, ya que no ven un buen manejo y distribución de recursos.

La siguiente gráfica muestra el resultado de la respuesta a la pregunta: ¿Conoce el destino de los impuestos recaudados por el gobierno?

Gráfica 2: Percepción del destino de los impuestos

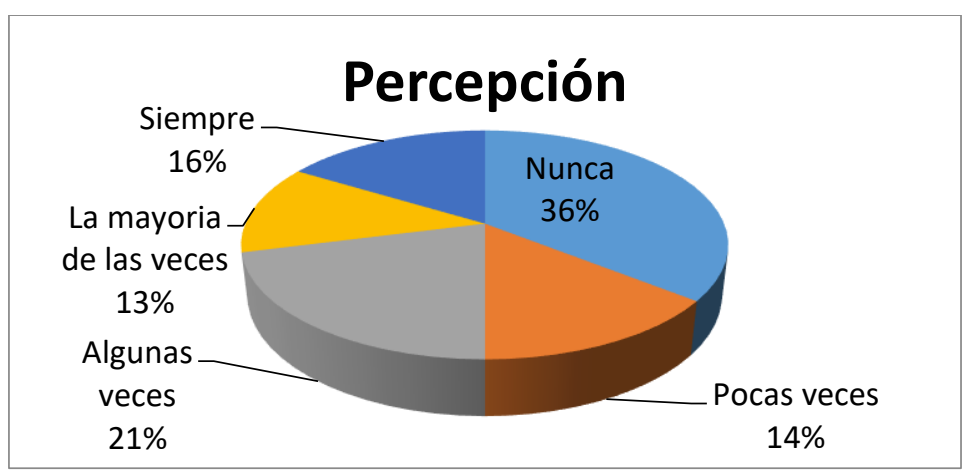

Fuente: Elaboración propia con datos del cuestionario para la evaluación de la cultura fiscal 


\section{Cultura tributaria en Hermosillo, Sonora, México}

Año 11, Número 21 julio-diciembre de 2019

María del Carmen Morales Tostado, Leivy Gisel Echeverría Rodríguez,

Beatriz Elena Huerta Urquijo, Erika Lorena Quinto Lorenzo

La mayoría de los contribuyentes desconoce el destino de los impuestos recaudados por el gobierno, ya que entre nunca, pocas veces y algunas veces se va a un $71 \%$.En un sentido más amplio, la percepción de que el gobierno no cumple con su parte de crear y mantener un ambiente que propicie el desarrollo pleno de los ciudadanos, afecta en gran medida la decisión de contribuir de manera regular y oportuna con el gasto público. De igual forma, cuando la idea de que el gasto público no llega a quienes más lo necesitan o es utilizado para otros fines y con ello fomentar la corrupción.

La siguiente gráfica muestra el resultado de la respuesta a la pregunta: ¿Usted considera que pagar sus impuestos ayuda a mejorar la calidad de los servicios públicos?

Gráfica 3: Actitudes para cumplir con los impuestos

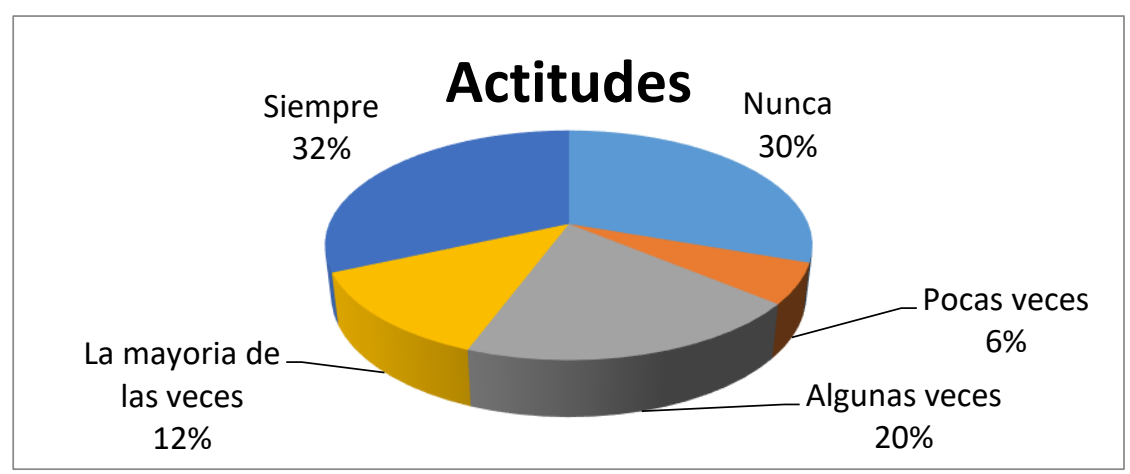

Fuente: Elaboración propia con datos del cuestionario para la evaluación de la cultura fiscal

En la gráfica, se muestra que un alto porcentaje considera que pagar sus impuestos ayuda a mejorar la calidad de los servicios público y cumple con sus obligaciones fiscales. Un $56 \%$ considera que nunca, pocas veces y algunas veces mejora la calidad de los servicios que ofrece el gobierno, un 44 $\%$ considera que si cumple con el pago de impuestos los servicios públicos que recibe son de calidad.Es primordial que el ciudadano vea que al cumplir con sus obligaciones fiscales y realice su contribución los servicios públicos que recibe sea presten con calidad y de manera oportuna. 
La siguiente gráfica muestra el resultado de la respuesta a la pregunta: ¿Considera usted que la educación fiscal puede generar conciencia para que todos los ciudadanos cumplan con sus obligaciones fiscales?

Gráfica 4: Educación Fiscal

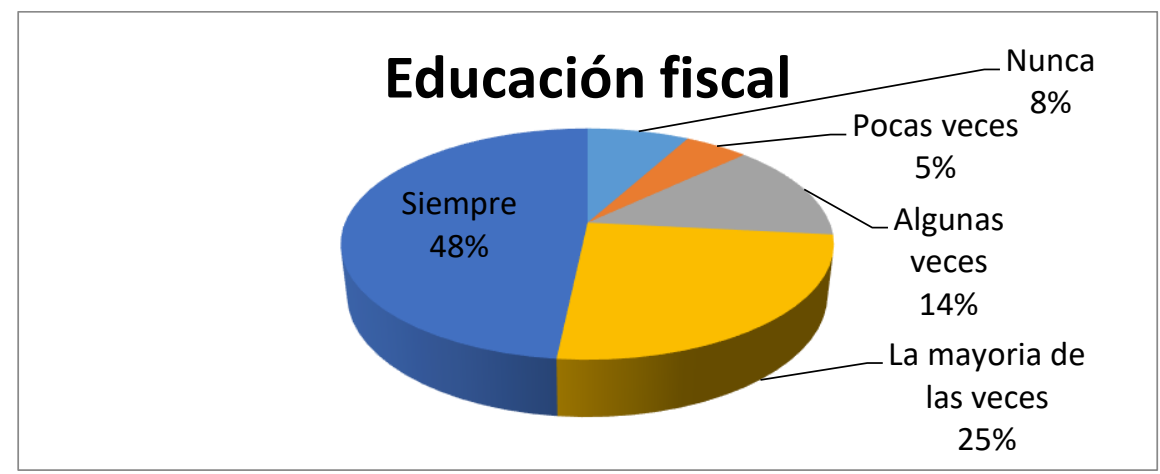

Fuente: Elaboración propia con datos del cuestionario para la evaluación de la cultura fiscal

Es importante destacar que un $73 \%$ de los encuestados coincide que la educación fiscal puede generar conciencia entre los ciudadanos para que cumplan con sus obligaciones fiscales.

La siguiente gráfica muestra el resultado de la respuesta a la pregunta ¿Con que frecuencia se atrasa en sus declaraciones?

\section{Gráfica 5: Educación Fiscal}

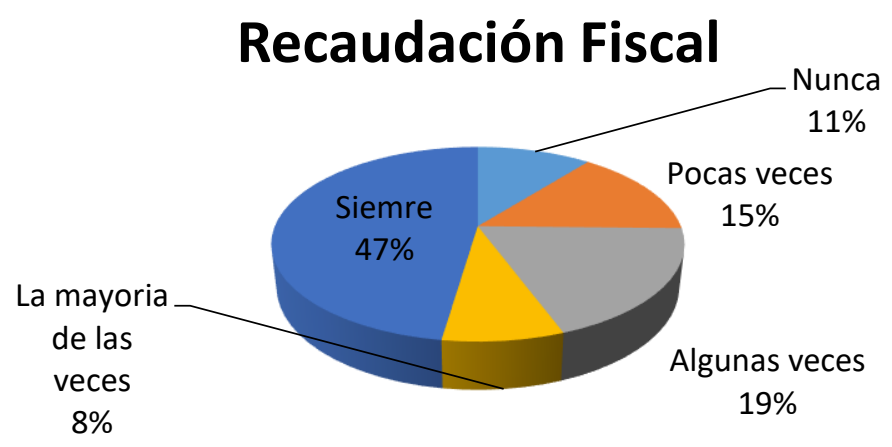

Fuente: Elaboración propia con datos del cuestionario para la evaluación de la cultura fiscal

Según los contribuyentes encuestados se muestra que la mayoría presenta sus declaraciones fiscales fuera de tiempo, en caso de que las 


\section{Cultura tributaria en Hermosillo, Sonora, México}

Año 11, Número 21 julio-diciembre de 2019

María del Carmen Morales Tostado, Leivy Gisel Echeverría Rodríguez, Beatriz Elena Huerta Urquijo, Erika Lorena Quinto Lorenzo presente, ya que una gran parte se muestra desmotivado ante el pago de impuestos y se ven influenciados por su entorno.

\section{CONCLUSIONES}

La evasión de impuestos es uno de los principales problemas que afectan la economía del país y aunque se han generado reformas que ayudan a su disminución, sigue existiendo un esquema muy utilizado por los contribuyentes para evadir el pago de impuestos.

La desconfianza y poca legitimidad en las autoridades de gobierno, además del desconocimiento y escasa cultura tributaria, hacen que nuestra sociedad tenga una actitud apática en cuanto al tema de los impuestos y esto repercute en la recaudación y distribución de los ingresos, lo cual se convierte en un círculo vicioso en el actuar de la ciudadanía.

Así mismo, en algunos casos existe la irresponsabilidad de simplemente no querer desempeñar las tareas gubernamentales de manera correcta, lo anterior debido a la facilidad con la que se manipulan las leyes, tal y como ocurrió con la llama estafa maestra, donde estuvieron implicados más de dos actores (comprador- vendedor) con lo que se engendró una de los robos más inverosímiles, con la venta de facturas por trabajos que nunca se llevaron a cabo. Lo anterior fue cometido no por una omisión o ignorancia de la ley, sino con todo el dolo que implica conocer la misma y vulnerarla.

En Hermosillo, Sonora. México, se tiene un vago conocimiento sobre lo que realmente representa tener cultura tributaria, por lo tanto, la ciudadanía no es consciente de la importancia y la gravedad de lo que implica no tener una cultura tributaria.

Las autoridades competentes deben ajustar las medidas preventivas y de castigo para quienes no cumplan sus responsabilidades, en cuanto a las obligaciones fiscales de personas físicas y morales, el acato al marco jurídico promueve el apego al Estado de derecho. 
Sin embargo, una vez más se hace hincapié en la formación de una ciudadanía consciente de la importancia de una educación tributaria, desde edades muy tempranas.

La información que proporcionó la encuesta se puede resumir de la siguiente manera, demostró que alrededor del $69 \%$ cuenta con conocimientos mínimos e inclusive nulos con relación a los impuestos que están obligados a contribuir y las sanciones a las que pueden incurrir en caso de no hacerlo, así como sus obligaciones y derechos fiscales como contribuyente.

En cuanto a las percepciones acerca del recaudo de los impuestos y el común ejercicio de las personas a pagar sus impuestos el resultado arrojó que prácticamente el $50 \%$ de los contribuyentes desconoce el destino de los impuestos recaudados por el gobierno y mencionaron que es habitual observar que los contribuyentes no paguen los impuestos correspondientes.

Según los contribuyentes encuestados el $73 \%$ presentan sus declaraciones fiscales fuera de tiempo, en caso de que las presente, ya que una gran parte se muestra desmotivado ante el pago de impuestos y se ven influenciados por su entorno.

Son muchos los esfuerzos que se han hecho para el cumplimiento de las leyes fiscales, a pesar de que estas, no son muy claras. 


\section{Cultura tributaria en Hermosillo, Sonora, México}

Año 11, Número 21 julio-diciembre de 2019

María del Carmen Morales Tostado, Leivy Gisel Echeverría Rodríguez, Beatriz Elena Huerta Urquijo, Erika Lorena Quinto Lorenzo

\section{REFERENCIAS}

CONSTITUCIÓN POLÍTICA DE LOS ESTADOS UNIDOS MEXICANOS.

México: Cámara de Diputados H. Congreso de Unión 2019

CÓDIGO FISCAL DE LA FEDERACIÓN. 2019. México: Cámara de Diputados del $\mathrm{H}$. Congreso de la Unión.

ESCALANTE, Gustavo,La Cultura Tributaria de los Contribuyentes, en Revista del Instituto de Especialización para Ejecutivos, mayo,2015

FLORES, Leonor, México, sin educación fiscal: OCDE. El Universal, 28 de julio, sección Economía,2015

GOBIERNO DE MÉXICO, Ver_ Secretaria de Hacienda y Crédito Público. Régimen de Incorporación Fiscal. http://omawww.sat.gob.mx/RegimenDelncorporacionFiscal/beneficios. htm (consultada el 15 de octubre de 2019).

MARGÁIN MANAUTOU, Emilio, Nociones de Política Fiscal, 2ª . Ed., México, Porrúa, 1998

NIETO DUEÑAS, Salvador, La educación tributaria como solución a la baja recaudación fiscal en México. Tesis de Licenciatura, Universidad de Guanajuato, 2003.

SECRETARIA DE HACIENDA Y CRÉDITO PÚBLICO. Programa de Apoyo a Deudores.

http://www.hacienda.gob.mx/politica_programa_apoyo/html/proafi3.pdf (consultada el 30 de septiembre de 2019).

SECRETARÍA DE HACIENDA Y CRÉDITO PÚBLICO. Régimen de Incorporación Fiscal y beneficios de Crezcamos Juntos. https://www.gob.mx/cms/uploads/attachment/file/362852/03-

_Re_gimen_de_incorporacio_n_fiscal.pdf (consultada el 01 de octubre de 2019). 


\section{SERVICIO DE ADMINISTRACIÓN TRIBUTARIA.}

http://132.248.9.34/hevila/Trimestrefiscal/2011/no96/5.pdf (consultada el 25 de septiembre de 2019).

\section{SERVICIO DE ADMINISTRACIÓN TRIBUTARIA.}

http://omawww.sat.gob.mx/Sumate/Paginas/default.htm\#Servicios (consultada el 15 de octubre de 2019).

SUPREMA CORTE DE JUSTICIA DE LA NACIÓN. Semanario Judicial de la Federación. Tomo XX noviembre.Tesis- 232309. PROPORCIONALIDAD Y EQUIDAD TRIBUTARIAS ESTABLECIDAS EN EL ARTICULO 31, FRACCION IV, CONSTITUCIONAL (Semanario Judicial de la Federación) Volúmenes 187-192, página 79. Amparo en revisión 2502/83 , 2004.

Recibido 8 de Agosto de 2019 Aceptado 7 de Noviembre de 2019 


\section{Cultura tributaria en Hermosillo, Sonora, México}

Año 11, Número 21 julio-diciembre de 2019

María del Carmen Morales Tostado, Leivy Gisel Echeverría Rodríguez, Beatriz Elena Huerta Urquijo, Erika Lorena Quinto Lorenzo

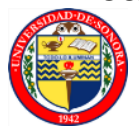

\section{ANEXO 1 \\ "UNIVERSIDAD DE SONORA" \\ DIVISIÓN DE CIENCIAS ECONÓMICAS Y ADMINISTRATIVAS C.E. 2018-2}

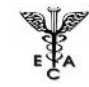

Estimado participante:

Le agradecemos anticipadamente por su colaboración. La siguiente encuesta es realizada con la finalidad de "Determinar la cultura fiscal de los contribuyentes en Hermosillo Sonora".

Marque con una "X" la opción que se adecue a su criterio.

\section{DATOS GENERALES}

1.1. Género:

a) Femenino ( ) b) masculino ( )

1.2. Edad:

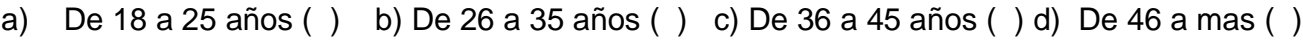

1.3. Nivel de Estudios:

a) Primaria ( ) b) Secundaria ( ) c) Bachillerato ( ) d) Licenciatura ( ) e) Maestría ( ) f) Doctorado ( )

Para evaluar las variables, marcar con una "X" en el casillero de su preferencia, utilice la siguiente escala.

\begin{tabular}{|c|c|c|c|c|c|c|c|c|c|}
\hline \multirow{2}{*}{\multicolumn{2}{|c|}{1}} & \multirow{2}{*}{\begin{tabular}{|c|}
$\mathbf{2}$ \\
Pocas veces
\end{tabular}} & \multirow{2}{*}{$\frac{3}{\text { Algunas veces }}$} & \multirow{3}{*}{\begin{tabular}{|c|}
$\mathbf{4}$ \\
La mayoría de las veces
\end{tabular}} & \multirow{2}{*}{\multicolumn{5}{|c|}{$\begin{array}{c}\mathbf{5} \\
\text { Siempre }\end{array}$}} \\
\hline & & & & & & & & & \\
\hline \multirow[t]{2}{*}{ № } & \multicolumn{3}{|c|}{ I.- CULTURA FISCAL } & & 1 & 2 & 3 & 4 & 5 \\
\hline & \multicolumn{4}{|c|}{ CONOCIMIENTO } & & & & & \\
\hline 1 & \multicolumn{4}{|c|}{ ¿Conoce los impuestos que como ciudadano está obligado a cumplir? } & & & & & \\
\hline 2 & \multicolumn{4}{|c|}{$\begin{array}{l}\text { ¿Conoce las sanciones a las que puede estar afecto en caso que no pague sus } \\
\text { impuestos? }\end{array}$} & & & & & \\
\hline 3 & \multicolumn{4}{|c|}{ ¿Conoce usted sus derechos y obligaciones fiscales como contribuyente? } & & & & & \\
\hline & \multicolumn{4}{|c|}{ II. PERCEPCIONES } & & & & & \\
\hline 4 & \multicolumn{4}{|c|}{ ¿Conoces el destino de los impuestos recaudados por el gobierno? } & & & & & \\
\hline \multirow[t]{2}{*}{5} & \multicolumn{4}{|c|}{ ¿Es común para usted que las personas no paquen impuestos? } & & & & & \\
\hline & \multicolumn{4}{|c|}{ III. ACTITUDES } & & & & & \\
\hline 6 & \multicolumn{4}{|c|}{$\begin{array}{l}\text { ¿Usted considera que pagar sus impuestos ayuda a mejorar la calidad de los } \\
\text { servicios públicos? }\end{array}$} & & & & & \\
\hline 7 & \multicolumn{4}{|c|}{ ¿Cumple usted regularmente con sus obligaciones fiscales? } & & & & & \\
\hline 8 & \multicolumn{4}{|c|}{$\begin{array}{l}\text { ¿Considera usted que el contribuyente es responsable del pago de sus } \\
\text { impuestos? }\end{array}$} & & & & & \\
\hline & \multicolumn{4}{|c|}{ IV. EDUCACIÓN FISCAL } & & & & & \\
\hline 9 & \multicolumn{4}{|c|}{ ¿Considera usted que la educación fiscal puede generar una cultura fiscal? } & & & & & \\
\hline 10 & \multicolumn{4}{|c|}{$\begin{array}{l}\text { ¿Alguna vez ha asistido a charlas impartidas por el SAT, respecto a información } \\
\text { fiscal? }\end{array}$} & & & & & \\
\hline 11 & \multicolumn{4}{|c|}{$\begin{array}{l}\text { ¿Considera usted que una adecuada y permanente capacitación fiscal ayudara a } \\
\text { incrementar la cultura fiscal en el país? }\end{array}$} & & & & & \\
\hline 12 & \multicolumn{4}{|c|}{$\begin{array}{l}\text { ¿Para usted el gobierno realiza una eficiente administración de los impuestos que } \\
\text { recauda? }\end{array}$} & & & & & \\
\hline & \multicolumn{4}{|c|}{ V. RECAUDACIÓN FISCAL } & & & & & \\
\hline 13 & \multicolumn{4}{|c|}{ ¿Con que frecuencia considera usted que se atrasa en sus declaraciones? } & & & & & \\
\hline 14 & \multicolumn{4}{|c|}{$\begin{array}{l}\text { ¿Alguna vez se ha sentido desmotivado para cumplir con el pago de sus } \\
\text { impuestos? }\end{array}$} & & & & & \\
\hline 15 & \multicolumn{4}{|c|}{$\begin{array}{l}\text { ¿Si observa que en su entorno evaden impuestos, usted también trataría de } \\
\text { hacerlo? }\end{array}$} & & & & & \\
\hline 16 & \multicolumn{4}{|c|}{$\begin{array}{l}\text { ¿Cuándo asiste al SAT a realizar algún trámite, espera mucho tiempo haciendo fila } \\
\text { para ser atendido? }\end{array}$} & & & & & \\
\hline 17 & $\begin{array}{l}\text { ¿Los } \mathrm{S} \\
\text { algún }\end{array}$ & $\begin{array}{l}\text { dores públicos del } \ \\
\text { icio? }\end{array}$ & atienden de man & correcta cuando solicitas & & & & & \\
\hline
\end{tabular}

